Tropical Journal of Pharmaceutical Research April 2017; 16 (4): 925-930

ISSN: $1596-5996$ (print); 1596-9827 (electronic)

(C) Pharmacotherapy Group, Faculty of Pharmacy, University of Benin, Benin City, 300001 Nigeria.

All rights reserved.

Available online at http://www.tjpr.org

Original Research Article

http://dx.doi.org/10.4314/tjpr.v16i4.26

\title{
Clinical effect of combined ulinastatin and continuous renal replacement therapy on management of severe sepsis with acute kidney injury
}

\author{
Qiying Fang ${ }^{1 \star}$ and Xinghua Zhao ${ }^{2}$ \\ ${ }^{1}$ Blood Purification Room, ${ }^{2}$ Department of Urology, The Second Affiliated Hospital of Zhengzhou University, Zhengzhou, China
}

*For correspondence: Email: jjb763@163.com; Tel: +86-0371-63934118.

Received: 15 January $2017 \quad$ Revised accepted: 24 March 2017

\begin{abstract}
Purpose: To explore the effect of a combination of ulinastatin and continuous renal replacement therapy (CRRT) for the treatment of severe sepsis with acute kidney injury (SAKI).

Methods: Clinical data for 106 patients diagnosed with SAKI from April 2013 to May 2015 in the intensive care unit (ICU) of Affiliated Hospital of Zhengzhou University, Zhengzhou, China, were collected and randomly assigned into two groups: treatment and control groups. The general information on the two groups were comparable. All the patients received a comprehensive treatment program which included fluid resuscitation, antibiotics, nutritional support and CRRT. The patients in treatment group also received intravenous injection of 300 thousand units $/ 10 \mathrm{~mL}$ ulinastatin thrice daily for 5 days. Serum levels of inflammatory cytokines, oxidative stress level, kidney and blood coagulation functions were assayed before and after treatment, using standard methodologies. In addition, adverse reactions and 28-day mortality were recorded.

Results: Levels of interleukin-6 (IL-6), tumor necrosis factor $\alpha$ (TNFa), serum C-reactive protein (CRP), procalcitonin (PCT), malondialdehyde (MDA), cystatin, blood urea nitrogen (BUN), prothrombin time $(P T)$, activated partial thromboplastin time (APTT), serum creatinine (SCr) and urinary kidney injury molecule-1 (Kim-1) in the treatment group were significantly lower than those in the control group after ulinastatin treatment $(p<0.05)$. However, superoxide dismutase (SOD), fibrinogen (Fib) and total antioxidant capacity $(T-A O C)$ in the treatment group were significantly higher in the control group after treatment $(p<0.05)$. No serious adverse drug reactions were seen in the two groups. In addition, there were no significant differences in 28-day mortality between the two groups.

Conclusion: These results suggest that ulinastatin combined with CRRT effectively decreases serum levels of inflammation in SAKI patients through a mechanism involving improvement of antioxidant capacity, reduction of oxidative stress, and enhancement of renal and coagulation functions.
\end{abstract}

Keywords: Ulinastatin, Continuous renal replacement therapy, Sepsis, Acute kidney injury, Inflammatory cytokines

Tropical Journal of Pharmaceutical Research is indexed by Science Citation Index (SciSearch), Scopus, International Pharmaceutical Abstract, Chemical Abstracts, Embase, Index Copernicus, EBSCO, African Index Medicus, JournalSeek, Journal Citation Reports/Science Edition, Directory of Open Access Journals (DOAJ), African Journal Online, Bioline International, Open-J-Gate and Pharmacy Abstracts

\section{INTRODUCTION}

Sepsis is a common complication of wounds, burns, infection and other severe conditions in clinics. Sepsis may lead to multiple organ dysfunction syndromes (MODS), a crucial area in intensive medicine. Studies show that there are about 18 million cases of new sepsis patients each year, accounting for $0.3 \%$ of the total population, and growing at a rate of $1.5 \%$ per year [1-4]. Although the treatment program for sepsis is regularly optimized, there is always high 
mortality rate in these patients. Acute kidney injury $(\mathrm{AKI})$ is one of the common complications of sepsis. Indeed about $40 \%$ of sepsis eventually develops into sepsis-mediated acute kidney injury (SAKI). Mortality from SAKI is up to $70 \%$, which is about twice the population of sepsis patients without $\mathrm{AKI}[\underline{5}, \underline{6}]$.

Continuous renal replacement therapy (CRRT) refers to blood purification therapy involving slow but continuous water clearance and solute exchange through diffusion, convection and adsorption. This has been widely used for clinical treatment of sepsis, severe acute pancreatitis and intractable heart failure $[\underline{7}, \underline{8}]$. In a retrospective study, Zhiping et al found that CRRT improved negative fluid balance in SAKI patients, reversed poor prognosis, enhanced recovery of renal function and reduced long-term mortality []․

Ulinastatin, a urinary tripsin inhibitor, improves microcirculation and tissue perfusion, relieves tissue and organ damage, regulates inflammatory cytokines and controls imbalances in inflammatory response, all of which contribute to efficacy and safety of treatment of sepsis, severe pancreatitis and acute coronary syndrome [10].

In this study, the clinical effects of treatment of SAKI with a combination of ulinastatin and CRRT were investigated.

\section{METHODS}

\section{Patients and study design}

The clinic data for 153 patients diagnosed with sepsis from April 2013 to May2015 in the Intensive Care Unit of The Second Affiliated Hospital of Zhengzhou University were collected. The recruitment criteria were (1) Age > 18 years; (2) patient must meet AKI diagnostic criteria as defined by the 2001 Washington International Conference (3) diagnosis of AKI must be consistent with AKI diagnostic criteria by the Acute Kidney Injury Collaboration Network in 2005 and (4) expected survival time of $>1$. The exclusion criteria were (1) medical history of any pre-existing chronic kidney disease such as glomerulonephritis and interstitial nephritis; (2) pre-existing kidney surgical history; (3) coexisting autoimmune disease, immunodeficiency disease or blood disorders; (4) patients who took glucocorticoids, immune suppressants and anticoagulants within the previous three months; (5) evidence of presence of CRRT or contraindications for ulinastatin application and (6) patients with severe liver and kidney dysfunction, and heart failure. Altogether, 106 patients met the required recruitment standards. These patients were assigned to observation and control groups using random list method. .This research was approved by the Ethical Committee of The Second Affiliated Hospital of Zhengzhou University (approval no.2016-02-01) and the studies were carried out according to Declaration of Helsinki promulgated in 1964 as amended in 1996 [11].

\section{Treatments}

All patients were treated for primary diseases, and given fluid resuscitation and antibiotics. Other procedures used included monitoring of vital signs, nutritional support, mechanical ventilation, correction of water and electrolyte acid-base balance disorders, prevention of heart failure, and control of blood sugar and blood pressure. On the basis of the above, treatment for control group was CRRT, wherein the blood filter was Prismaflex (Swedish Campbell Medical Products Co., Ltd.), the colander was AN69M100 matched with the blood filter; vascular access was large-diameter double lumen intravenous catheter for femoral vein indwelling for blood purification with mode of continuous veno - venous hemofiltration (CVVH, a mode of CRRT), the replacement fluid formulation was provided by Nanjing Military Region General hospital, replacement fluid flow rate was 35 $\mathrm{ml} \cdot \mathrm{kg}^{-1} \cdot \mathrm{h}^{-1}$ and blood flow rate was $200 \mathrm{ml} / \mathrm{min}$, lasting $12 \mathrm{~h} \sim 24 \mathrm{~h}$ each time. In the absence of contra-indications, conventional low molecular weight heparin was applied continuously for 5 days. The observation group received intravenous injection of $300,000 \mathrm{U}$ of ulinastatin (Guangdong Techpool Pharmaceutical Co., Ltd., Zhunzi H20040506, China) continuously 3 times a day for 5 days.

\section{Evaluation of serum parameters}

Prior to treatment and 7 days after treatment, serum CRP, PCT, TNF- $\alpha$ and IL- 6 levels were assayed by immune nephelometry, electrochemical luminescence and enzyme-linked immunosorbent assay (ELISA) kits, as appropriate. The kits were purchased from Roche Co., Ltd., Germany. Other parameters that were assayed before and after 7-day treatment were BUN, SCr, urinary Kim-1 and cystatin (CysC). The last two parameters were measured by ELISA assay (Shanghai Haibo Institute Co., Ltd, Shanghai China). Prothrombin time (PT), APTT, Fib, SOD, T-AOC and MDA levels were determined with appropriate kits using OLYMPUS Automatic Biochemistry Instrument (Nanjing Jiancheng Bioengineering 
Institute, Nanjing, China). Mortality and adverse reactions were monitored over a period of 28 days.

\section{Statistics analysis}

Experimental data were analyzed statistically with SPSS 20.0 software. Measurement data were expressed as mean \pm standard deviation (SD). Comparison between the two groups was conducted with analysis of variance (ANOVA). Enumeration data were expressed as percentage, and compared with Chi-square test. $P<0.05$ was considered statistically significant.

\section{RESULTS}

\section{Characteristics of participants}

Each group had 53 patients. Four patients in treatment group withdrew from the study within 7 days due to transfers and other factors, while 6 individuals withdrew from control group. Thus the actual treatment cases were 49 in the treatment group and 47 in control.

There was no statistically significant differences in gender, age, primary tumor site, pathogens, acute physiology and chronic health evaluation (APACE II rates) and Marshall score comparison between the two groups $(p>0.05)$ (Table 1$)$.

\section{Inflammatory factors}

Before treatment, there were no significant differences in levels of serum PCT, CRP, IL-6 and TNF- $\alpha$ between the two groups $(p>0.05)$. However, after treatment with ulinastatin, serum levels of PCT, CRP, IL- 6 and TNF- $\alpha$ decreased significantly when compared with values before the treatment $(p<0.05)$. Importantly, after treatment, levels of serum PCT, CRP, IL-6 and TNF- $\alpha$ in the observation group significantly decreased relative to corresponding levels in control $(p<0.05$, Table 2$)$.

\section{Renal function}

Before treatment, there were no significant differences in levels of serum CysC, BUN, SCr and urinary Kim-1 between patients in the two groups. However, after treatment, serum CysC, BUN, SCr and urinary Kim-1 levels were significantly increased when compared with corresponding values before treatment, although the post-treatment levels in the observation group decreased more than in the control group $(p<0.05$, Table 3).

\section{Effect on coagulation function}

No significant differences existed in the levels of PT, APTT and serum Fib in patients from the two groups prior to treatment.

Table 1: Comparison of baseline characteristics of the two groups

\begin{tabular}{|c|c|c|c|c|c|c|c|c|c|c|c|c|}
\hline \multirow{2}{*}{ Group } & \multirow{2}{*}{$\mathbf{N}$} & \multirow{2}{*}{ Age } & \multirow{2}{*}{$\begin{array}{c}\text { Gender } \\
\text { (M / F) }\end{array}$} & \multicolumn{4}{|c|}{ Primary lesion site } & \multicolumn{3}{|c|}{ Pathogenic bacteria } & \multirow{2}{*}{ APACE II } & \multirow{2}{*}{ Marshall } \\
\hline & & & & $L$ & $A$ & $B$ & 0 & GN & GP & $M I$ & & \\
\hline Observation & 49 & $56.7 \pm 12.5$ & $35 / 14$ & 22 & 14 & 7 & 6 & 34 & 8 & 7 & $22.3 \pm 4.5$ & $10.1 \pm 2.5$ \\
\hline Control & 47 & $59.3 \pm 11.6$ & $30 / 17$ & 25 & 9 & 10 & 3 & 37 & 6 & 3 & $21.7 \pm 3.9$ & $10.6 \pm 2.1$ \\
\hline$t / x^{2}$ & & 1.07 & 0.63 & \multicolumn{4}{|c|}{2.77} & \multirow{2}{*}{\multicolumn{3}{|c|}{$\begin{array}{l}1.92 \\
0.38\end{array}$}} & 0.70 & 1.07 \\
\hline$P$ & & 0.29 & 0.43 & & & & & & & & 0.48 & 0.29 \\
\hline
\end{tabular}

$M=$ male; $F=$ female; $L=$ lung; $A=$ abdominal cavity; $B=$ blood; $O=$ others; $G N=$ gram negative bacteria; $G P=$ Gram positive bacteria; $M I=$ mixed infection

Table 2: Levels of serum inflammatory cytokines in the two groups before and after treatment with ulinastatin

\begin{tabular}{|c|c|c|c|c|c|c|c|c|}
\hline \multirow{2}{*}{ Group } & \multicolumn{2}{|c|}{ PCT (ng/mL) } & \multicolumn{2}{|c|}{ CRP (mg/L) } & \multicolumn{2}{|c|}{ TNF- $\alpha$ (ng/L) } & \multicolumn{2}{|c|}{ IL-6 (ng/L) } \\
\hline & Before & After & Before & After & Before & After & Before & After \\
\hline Treatment & $5.5 \pm 2.2$ & $1.6 \pm 0.9^{*} \#$ & $75.5 \pm 21.3$ & $27.4 \pm 13.3^{* \#}$ & $177.5 \pm 25.9$ & $107.3 \pm 20.7^{* \#}$ & $213.2 \pm 27.7$ & $127.5 \pm 24.7^{* \#)}$ \\
\hline Control & $5.7 \pm 2.2$ & $2.7 \pm 0.8$ & $73.6 \pm 18.8$ & $48.7 \pm 14.6$ & $183.3 \pm 23.8$ & $138.4 \pm 26.8^{\circ}$ & $209.3 \pm 31.65$ & $159.7 \pm 28.2$ \\
\hline$t$ & 0.32 & 6.24 & 0.48 & 7.57 & 1.15 & 6.42 & 0.70 & 6.03 \\
\hline$P$ & 0.75 & 0.00 & 0.63 & 0.00 & 0.25 & 0.00 & 0.49 & 0.00 \\
\hline
\end{tabular}

Table 3: Levels of renal function parameters in the two groups before and after treatment with ulinastatin

\begin{tabular}{lcccccccc}
\hline \multirow{2}{*}{ Group } & \multicolumn{2}{c}{ Urinary Kim-1 $(\boldsymbol{\mu g} / \mathbf{L})$} & \multicolumn{2}{c}{ Cys C $(\mathbf{m g} / \mathbf{L})$} & \multicolumn{2}{c}{ BUN $(\mathbf{m m o l} / \mathbf{L})$} & \multicolumn{2}{c}{$\mathbf{S C r}(\boldsymbol{\mu m o l} / \mathbf{L})$} \\
\cline { 2 - 9 } & Before & After & Before & After & Before & After & Before & After \\
\hline Treatment & $4.75 \pm 0.38$ & 5.2 & $3.32 \pm 0.36$ & $5.28 \pm 0.47^{\#}$ & $11.32 \pm 3.34$ & $14.53 \pm 3.15^{\#}$ & $137.37 \pm 22.54$ & $238.47 \pm 25.45^{*}$ \\
Control & $4.68 \pm 0.40$ & $5.72 \pm 0.35$ & $3.40 \pm 0.28$ & $6.11 \pm 0.42$ & $10.87 \pm 3.58$ & $16.42 \pm 2.77^{*}$ & $133.82 \pm 20.73$ & $186.62 \pm 27.83^{\circ}$ \\
$t$ & 0.89 & 5.80 & 1.22 & 9.21 & 0.64 & 3.15 & 0.81 & 9.87 \\
$P$ & 0.38 & 0.00 & 0.22 & 0.00 & 0.52 & 0.00 & 0.42 & 0.00 \\
\hline \multicolumn{2}{r}{${ }^{*} p<0.05$ compared with pre- treatment; \#p < 0.05 compared with control group } & &
\end{tabular}

${ }^{*} p<0.05$ compared with pre- treatment; $\# p<0.05$ compared with control group 
However, after treatment, PT and APTT levels were significantly reduced relative to values before treatment, while serum Fib level became significantly elevated $(p<0.05)$. After treatment, PT and APTT levels in the observation group were significantly lower than corresponding values in the control group $(p<0.05)$, while serum Fib level was significantly higher $(p<$ 0.05 , Table 4).

\section{Oxidative stress}

Prior to treatment, there were no significant differences in serum SOD, AOC and MDA profiles between patients in the two groups, but after treatment, SOD and $A O C$ increased significantly, while MDA was significantly reduced $(p<0.05)$.

In addition, SOD and AOC levels in observation group were significantly higher than corresponding values in the control group, while serum MDA was significantly reduced $(p<0.05$, Table 5).

\section{Mortality and adverse reactions}

During the ulinastatin treatment, there were no serious adverse drug reactions in either group. In the observation group, one patient had rashes, another one had mild drop in blood pressure while one patient experienced paroxysmal atrial fibrillation. Two cases of mild nausea were seen in the control group. These adverse conditions were relieved after symptomatic treatment. During the 28-day treatment, there were 13 dead cases in the observation group (mortality rate of $17.8 \%$ ), while 17 cases died in the control group (mortality rate of $20.2 \%$ ), indicating no significant difference in mortality in the two groups $\left(x^{2}=\right.$ $0.15, p=0.70$ ).

\section{DISCUSSION}

Mechanisms involved in sepsis-mediated AKI are complicated. It is thought that toxins released by infections activate inflammatory cells to induce large release of inflammatory mediators such as IL-6, TNF-a.

These activate Toll-like 4 receptor, and form inflammatory reaction cascade leading to pathophysiological changes in renal vascular endothelial cells and renal parenchymal cells, and eventually kidney damage [12]. Sepsis patients have changes in their neuroendocrine system such as activation of sympathetic nervous system, release of vasopressin, and activation of renin - angiotensin - aldosterone system. These changes can result in decrease in renal blood flow, renal tubular epithelial cell necrosis and decline in renal function [13].

Release of large number of inflammatory factors can damage the endothelial cells of patients with sepsis, activate the coagulation system to a hyper-coagulable state, induce formation of renal micro vascular thrombosis and affect renal perfusion [14]. In addition, endotoxemia increases reactive oxygen species (ROS) generated by mitochondrial respiratory chain and decreases enzyme activities in tubular epithelial cell. These will induce retention of large amounts of oxygen free radicals in the body, and then accelerate lipid peroxidation, membrane damage, apoptosis and renal tubular epithelial damage and decline in kidney function [15].

Table 4: Coagulation function of the two groups before and after treatment

\begin{tabular}{lcccccc}
\hline \multirow{2}{*}{ Group } & \multicolumn{2}{c}{ PT $(\mathbf{s})$} & \multicolumn{2}{c}{ APTT $(\mathbf{s})$} & \multicolumn{2}{c}{ Fib (g/L) } \\
\cline { 2 - 6 } & Before & After & Before & After & Before & After \\
\hline Treatment & $23.15 \pm 1.52$ & $14.42 \pm 1.50^{*} \#$ & $47.87 \pm 4.12$ & $41.28 \pm 4.37^{* \#}$ & $1.77 \pm 0.52$ & $2.93 \pm 0.63^{*}{ }^{*}$ \\
Control & $22.82 \pm 1.35$ & $16.35 \pm 1.47^{*}$ & $48.10 \pm 4.25$ & $44.57 \pm 4.56^{*}$ & $1.82 \pm 0.59$ & $2.12 \pm 0.57^{*}$ \\
$T$ & 1.13 & 6.43 & 0.27 & 3.65 & 0.45 & 6.67 \\
$P$ & 0.26 & 0.00 & 0.79 & 0.00 & 0.66 & 0.00 \\
\hline
\end{tabular}

${ }^{*} p<0.05$ compared with pre- treatment; \#p < 0.05 compared with control group

Table 5: Oxidative stress status of the two groups before and after treatment with ulinastatin

\begin{tabular}{|c|c|c|c|c|c|c|}
\hline \multirow{2}{*}{ Group } & \multicolumn{2}{|c|}{ SOD (U/mL) } & \multicolumn{2}{|c|}{ T-AOC (U/mL) } & \multicolumn{2}{|c|}{ MDA (nmol/mL) } \\
\hline & Before & After & Before & After & Before & After \\
\hline Observation & $66.36 \pm 12.35$ & $86.46 \pm 11.73^{* \#}$ & $5.35 \pm 1.12$ & $7.65 \pm 1.20^{\#}$ & $14.35 \pm 1.10$ & $10.54 \pm 1.17^{* \#}$ \\
\hline Control & $67.42 \pm 10.68$ & $75.34 \pm 12.55$ & $5.42 \pm 1.23$ & $6.33 \pm 1.05$ & $14.42 \pm 1.13$ & $12.20 \pm 1.08$ \\
\hline $\mathrm{t}$ & 0.45 & 4.76 & 0.29 & 5.79 & 0.31 & 7..29 \\
\hline$P$ & 0.65 & 0.00 & 0.77 & 0.00 & 0.76 & 0.00 \\
\hline
\end{tabular}


The key to SAKI treatment is to remove endotoxins in patients, block inflammation, reduce oxidative stress levels, improve blood coagulation, and gradually enhance renal function. CRRT can maintain stable dynamics of blood flow in patients with sepsis, remove metabolic waste, endotoxins and inflammatory cytokines and correct water and electrolyte acidbase disorders. In a prospective study, Xu et al found that early CRRT greatly improved 28-day survival in patients with SAKI [16]. Dai et al also confirmed that CRRT can significantly improve the prognosis of patients with SAKI, and shorten organ support time and length of stay in ICU [17].

Ulinastatin, as a broad-spectrum proteinase inhibitor, inhibits all types of hydrolytic enzymes, stabilize lysosomal membrane, reduce oxygen free radicals, suppress the release of inflammatory mediators, and also mitigate damage to heart, kidney, brain, lung and other organs. Wu et al found that ulinastatin can effectively reduce the expression of regulatory $T$ cells (Treg) and helper T- cells17 (Th17) in patients with severe sepsis, reverse imbalance in Treg/Th 17 ratio, down-regulate IL-17, IL-6, and IL-10 levels; increase monocyte human leukocyte DR antigen (HLA-DR) and improve cellular immunity, all of which are expected to improve the prognosis of patients with severe sepsis [18].

This study showed that serum PCT, CRP, IL-6 and TNF- $\alpha$ levels in the observation group were significantly lower than those of the control group after treatment with ulinastatin, suggesting that the drug can control inflammatory responses by reducing levels of inflammatory factors in patients with sepsis, thereby relieving inflammatory damage to tissues and organs. Superoxide Dismutase (SOD) is major antioxidant enzyme for scavenging oxygen free radicals in patients with sepsis, and T-AOC is a comprehensive indicator reflecting the overall level of enzymatic and non-enzymatic antioxidants.

MDA is one of the main products of oxygen free radical-induced lipid peroxidation in biological membranes; it indicates indirectly the levels of oxygen free radicals and oxidative damage [19]. This study showed that serum SOD and AOC levels in the observation group were more remarkably increased than in control after treatment, while serum MDA level were significantly lower. This suggests that ulinastatin can effectively reduce oxygen free radical levels in patients with SAKI and elevate antioxidant capacity to reduce oxidative stress damage. The large number of endotoxins and inflammatory mediators in patients with SAKI can result in dysfunction of the endogenous and exogenous coagulation system, accompanied by declines in coagulation factors and platelets. Thus, PT and APTT in SAKI patients were increased significantly, while Fib level declined. The results of this study show PT and APTT in the observation group patients were significantly lower than in the control group after treatment, while serum Fib levels were significantly higher, indicating that ulinastatin can effectively improve blood coagulation in patients with SAKI. However, the specific regulatory mechanism involved in this ulinastatin effect remains to be further explored.

Kim-1 is a type I trans-membrane protein with high tissue-specificity, which has good sensitivity and specificity in the diagnosis of AKI [20]. CysC is a cysteine protease inhibitor which reflects changes in glomerular filtration rate [21]. The results of this study show that serum CysC, BUN, SCr and urinary Kim-1 in the observation group were significantly lower than in the control group after treatment, indicating that ulinastatin combined with CRRT can prevent continued deterioration of renal function in patients with SAKI. In addition, this study showed there were no treatment-related serious adverse reactions, suggesting that ulinastatin did not increase metabolic burden of SAKI patients or impose additional side effects.

\section{Limitations of the study}

There was no long term follow-up on the SAKI patients. There is still a need for a randomized controlled study with a larger sample size and multiple study centers to confirm these results.

\section{CONCLUSION}

Ulinastatin combined with CRRT effectively controls serum levels of inflammation factors in SAKI patients via improvement in antioxidant capacity, reduction in oxidative stress, as well as enhancement of renal and coagulation functions.

\section{DECLARATIONS}

\section{Acknowledgement}

None declared

\section{Conflict of Interest}

No conflict of interest associated with this work.

\section{Contribution of Authors}


The authors declare that this work was done by the authors named in this article and all liabilities pertaining to claims relating to the content of this article will be borne by them.

\section{Open Access}

This is an Open Access article that uses a funding model which does not charge readers or their institutions for access and distributed under the terms of the Creative Commons Attribution License (http://creativecommons.org/licenses/by/ 4.0) and the Budapest Open Access Initiative (http://www.budapestopenaccessinitiative.org/rea d), which permit unrestricted use, distribution, and reproduction in any medium, provided the original work is properly credited.

\section{REFERENCES}

1. Angus DC, Linde-Zwirble WT, Lidicker J, Clermont G, Carcillo J, Pinsky MR. Epidemiology of severe sepsis in the United States: analysis of incidence, outcome, and associated costs of care. Crit Care Med 2001; 29(7): 1303-1310.

2. Martin GS, Mannino DM, Eaton S, Moss M. The epidemiology of sepsis in the United States from 1979 through 2000. N Engl J Med 2003; 348(16): 1546-1554.

3. Dombrovskiy VY, Martin AA, Sunderram J, Paz HL. Rapid increase in hospitalization and mortality rates for severe sepsis in the United States: a trend analysis from 1993 to 2003. Crit Care Med 2007; 35(5): 1244-1250.

4. Yang $Y, X i e ~ J-F, Y u K-J$, Yao C, Li J-G, Guan X-D, Yan J, Ma X-C, Kang Y, Yang C-S. Epidemiological Study of Sepsis in China: Protocol of a Cross-sectional Survey. Chin Med J (Engl) 2016; 129(24): 2967.

5. Eswarappa M, Madhyastha PR, Puri S, Varma V, Bhandari A, Chennabassappa G. Postpartum acute kidney injury: a review of 99 cases. Ren Fail 2016; 38(6): 889-893.

6. Honore PM, Jacobs $R$, De Waele E, Diltoer M, Spapen $H D$. Renal blood flow and acute kidney injury in septic shock: an arduous conflict that smolders intrarenally? Kidney Int 2016; 90(1): 22-24.

7. Faubel S, Shah PB. Immediate consequences of acute kidney injury: the impact of traditional and nontraditional complications on mortality in acute kidney injury. Adv Chronic Kidney Dis 2016; 23(3): 179-185.

8. Gulla KM, Sachdev A, Gupta D, Gupta N, Anand K, Pruthi $P$. Continuous renal replacement therapy in children with severe sepsis and multiorgan dysfunctionA pilot study on timing of initiation. Indian J Crit Care Med 2015; 19(10): 613.
9. Sun Z, Sun F, Niu C, Shen X, Ye H, Cao H. Continuous renal replacement therapy and negative fluid balance improves renal function and prognosis of patients with acute kidney injury in sepsis. Zhonghua wei zhong bing ji jiu yi xue 2015; 27(5): 321-326.

10. Wang G, Wen J, Wilbur RR, Wen P, Zhou S-F, Xiao X. The effect of somatostatin, ulinastatin and Salvia miltiorrhiza on severe acute pancreatitis treatment. Am J Med Sci 2013; 346(5): 371-376.

11. Word Health Organization. Declaration of Helsinki. BMJ 1996; 313(7070): 1448-1449.

12. Karino $S$, Kaye $K S$, Navalkele B, Nishan B, Salim $M$, Solanki S, Pervaiz A, Tashtoush N, Shaikh H, Koppula S. Epidemiology of Acute Kidney Injury among Patients Receiving Concomitant Vancomycin and PiperacillinTazobactam: Opportunities for Antimicrobial Stewardship. Antimicrob Agents Chemother 2016; 60(6): 3743-3750.

13. Pynn JM, Parravicini E, Saiman L, Bateman DA, Barasch $J M$, Lorenz JM. Urinary neutrophil gelatinase-associated lipocalin: potential biomarker for late-onset sepsis. Pediatr Res 2015; 78(1): 76-81.

14. Verma SK, Molitoris BA. Renal endothelial injury and microvascular dysfunction in acute kidney injury. in Seminars in nephrology. 2015. Elsevier.

15. Zhou J, Li Y, Tang Y, Liu F, Yu S, Zhang L, Zeng X, Zhao $Y, F u P$. Effect of acute kidney injury on mortality and hospital stay in patient with severe acute pancreatitis. Nephrol 2015; 20(7): 485-491.

16. Xu T SX, Cui YP. The clinical study of CRRT in treatment of patients with sepsis acute kidney injury. Chin J Blood Purifi 2013; 12(12).

17. Dai T CS, Yang XL. Comparison of clinical efficacy of continuous renal replacement therapy and intermittent hemodialysis for sepsis acute kidney injury. Chin Crit Care Med 2016; 28(3).

18. Wu TJ ZL, Hang CC. The regulation effect of ulinastatin on inflammatory immune imbalance of patients with severe sepsis. Chin Crit Care Med 2013; 25(4).

19. $Y u$ YM HF, Zhang $X$. The relationship between hyperhomocysteinemia, oxidative stress and microinflammation in chronic renal failure patients and its role in atherosclerosis. Chin J Internal Med 2004; 43(4).

20. Zhang Z, Cai CX. Kidney injury molecule-1 (KIM-1) mediates renal epithelial cell repair via ERK MAPK signaling pathway. Mol Cell Biochem 2016; 416(1-2): 109-116.

21. Zappitelli M, Greenberg JH, Coca SG, Krawczeski CD, Li $S$, Thiessen-Philbrook HR, Bennett MR, Devarajan P, Parikh CR. Association of definition of acute kidney injury by cystatin $C$ rise with biomarkers and clinical outcomes in children undergoing cardiac surgery. JAMA Pediatrics 2015; 169(6): 583-591. 